\title{
Strength Analysis and Experiment of High Speed Railway Gearbox Bracket
}

\author{
Jianwei Yang ${ }^{*}, 1,2$, Minghan Yang ${ }^{1,2}, \mathrm{Xi} \mathrm{Li}^{3}$ and Xing Wang ${ }^{4}$
}

${ }^{1}$ School of Machine-Electricity and Automobile Engineering, Beijing University of Civil Engineering Architecture, Beijing, 100044, China

${ }^{2}$ Beijing Engineering Research Center of Monitoring for Construction Safety, Beijing University of Civil Engineering Architecture, Beijing, 100044, China

${ }^{3}$ Subway Operation Technology Centre, Mass Transit Railway Operation Corporation LTD, Beijing, 102208, China

${ }^{4}$ School of Computing, Taiyuan University of Science and Technology, Taiyuan, Shanxi, 030024, China

\begin{abstract}
Light-weighting of high speed railway equipments has become a major trend, which leads to the upgradations of equipments. A new C-shaped bracket has been produced to connect the driving gearbox with the bogie. This paper built a three-dimensions model of a $\mathrm{C}$-shaped bracket, got the maximum distribution of the stress and deformation under two different working conditions from finite element analysis (FEA). Then it presents a method to perform a corresponding experiment. It is observed that the computed values form FEA are in very good agreement with the experimental values, which both verified the structural strength of this C-shaped bracket.
\end{abstract}

Keywords: C-shaped bracket, Finite element analysis, High speed railway, Structural strength experiment.

\section{INTRODUCTION}

With the rapid and continuous development of China's high speed railway, transmission technology has turned decisive [1]. There are several methods, most of which use a suspender to connect the driving gearbox to the bogie. The $\mathrm{C}$ shaped bracket is one of the latest solutions for such connections while light-weighting the equipments in high speed railway [2]. Consequently, the structural strength of the bracket has become a major concern.

Finite-element analysis (FEA) is wildly used in stress analysis [3], but usually, due to the complexity of the loads and fixation, there is a scarcity of the corresponding experimental values to compare with the for the computed values from FEA.

This paper built a finite-element model of a C-shaped bracket, and compared the values from both FEA and the corresponding experiment, which is performed with customized holding devices, to provide the basis and direction for the light-weighting of high speed railway equipments.

\section{STRESS ANALYSIS OF C-SHAPED BRACKET}

\subsection{Finite-Element Model of C-Shaped Bracket}

There are three methods of modeling in a FEA software [4]:

*Address correspondence to this author at the School of Machine-electricity and Automobile Engineering, Beijing University of Civil Engineering Architecture, Beijing, 100044, P.R. China; Tel: +86 1068322515 ; E-mail: railyjw@163.com
(1) Model directly in the FEA software.

(2) Entity modeling.

(3) Use a Computer Aided Design (CAD) software.

In this paper, SolidWorks is used to build the threedimensions model of the $\mathrm{C}$-shaped bracket which as shown in Fig. (1). The model was saved as a IGES file, then imported to Ansys, an FEA software.

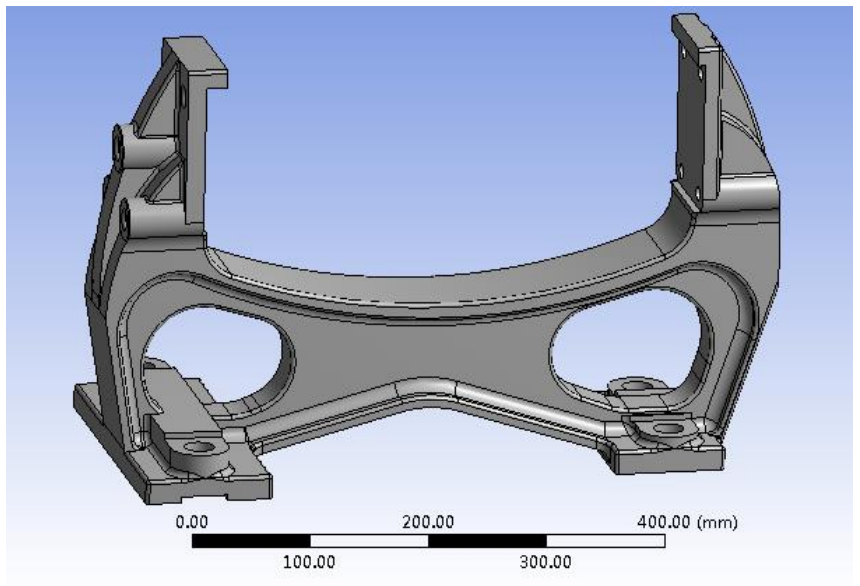

Fig. (1). Three-dimensional model of C-shaped bracket.

\subsection{Material Parameters}

The material of the bracket was applied, which is grade $\mathrm{E}$ cast steel, to the model. Table 1 lists it's Young's modulus, yield strength and Poisson's ratio. 
Table 1. Properties of grade $\mathbf{E}$ cast steel.

\begin{tabular}{|c|c|c|}
\hline Young's Modulus & Yield Strength & Poisson 's Ratio \\
\hline \hline $2.1 \mathrm{GPa}$ & $690 \mathrm{MPa}$ & 0.3 \\
\hline
\end{tabular}

\subsection{Boundary Conditions}

In Fig. (2), a C-shaped bracket was installed to a CRH3A driving gearbox which is able to operate at $250 \mathrm{~km}$ per hour. It also shows the loads during positive and reversed rotations. While operating, the bracket is fixed to the bogie by 4 bolts. There is a bumper block on each side where the bracket connects to the gearbox to absorb the shock. The vertical stiffness of the block is $6.6 \mathrm{kN} / \mathrm{mm}$, and the working stroke is $4 \sim 5 \mathrm{~mm}$.

To set up the boundary conditions, add fixed supports on the surfaces where the bracket contacts to the bogie by bolts, and 2 spring elements to simulate the bumper blocks.

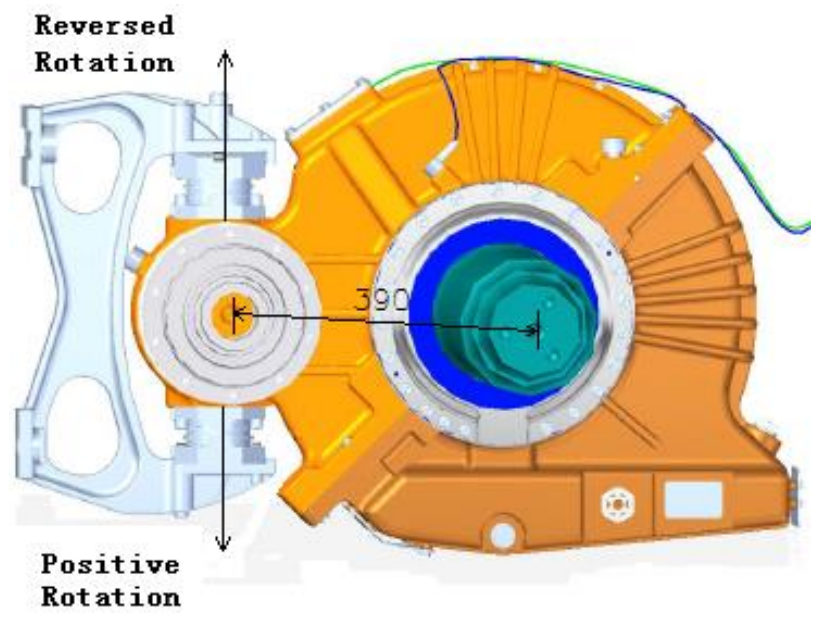

Fig. (2). C-shaped bracket attached to gearbox.

\subsection{Loading Definition}

Obviously, the severest working conditions of the Cshaped bracket is when under the short circuit torque. The load that the bracket sustained is

$F=\frac{M \quad(i+1)}{L}$

where,

$F$---the force to the bracket;

$M$---the short circuit torque;

$i$---the gear ratio;

$L$---the distance between the center of load and the wheel shaft.

The direction of this load is straight up during reversed rotation, and down if it's positive rotation. The gravity of the gearbox shared to the bracket is far less than this load, so can be ignored.

Table 2 lists the gear ratio, the distance between the center of load and the wheel shaft, and the short circuit torque of this transmission gear. Therefore, the load under the short circuit torque is $\pm 112 \mathrm{kN}$, which is also listed in Table 2 .

Table 2. Relevant factors of working condition.

\begin{tabular}{|c|c|c|c|}
\hline Gear Ratio & Distance & Short Circuit Torque & Load \\
\hline \hline 3.444 & $390 \mathrm{~mm}$ & $\pm 9840 \mathrm{Nm}$ & $\pm 112 \mathrm{kN}$ \\
\hline
\end{tabular}

\subsection{Meshing}

Two common methods of meshing are free and mapped meshing [3-6]. This paper uses mapped meshing, the mesh has 45665 nodes and 25670 elements.

\subsection{Results}

The stress nephogram of the bracket during positive and reversed rotations are given in Fig. (3), which shows the max stress of 281.7 MPa and 277.0 Mpa, both at the corner next to the loading point. Fig. (4) shows the total deformation.

\section{STRESS EXPERIMENT OF C-SHAPED BRACKET}

\subsection{Holding Devices and Experiment Method}

This paper presents a set of holding devices for the stress experiment as shown in Fig. (5). The hydraulic jack generates the extrusion force, which can be adjusted by the force sensors, to load the bracket fixed to the welded frame. A continuous data acquisition system (CDAS) [7] is used to record the signals from the $45^{\circ}$ strain rosettes attached to the danger points which are determined by the FEA results. Fig (6) shows the danger points of the bracket.

The holding devices are welded structures made of Q235 steel plates. To reach the $112 \mathrm{kN}$ load for both bracket, the hydraulic jack has to generate more than $224 \mathrm{kN}$ extrusion force. Fig. (7) shows the stress nephrogram with a max stress of 178.4 MPa, which is less then the yield strength of Q235 steel (235 MPa), the safety factor is 1.32 , satisfies the strength requirement.

This method can simulate two rotating modes under real conditions such as fixation and loads. Two brackets can be tested at the same time, and it's easy to perform.

\subsection{Experiment Procedures}

To perform the test, the following steps are taken:

- Install the brackets to the frame as design requires;

- Zero the sensors before being placed into position;

- Attach the $45^{\circ}$ strain rosettes to the danger points, connect them to the CDAS, start recording;

- Supercharge the hydraulic jack till the readings of the sensors reach $112 \mathrm{kN}$;

- $\quad$ Keep the load for 5 minutes, then unload;

- $\quad$ Repeat steps 4 and 5 for three times to get the mean value;

- Finish. 


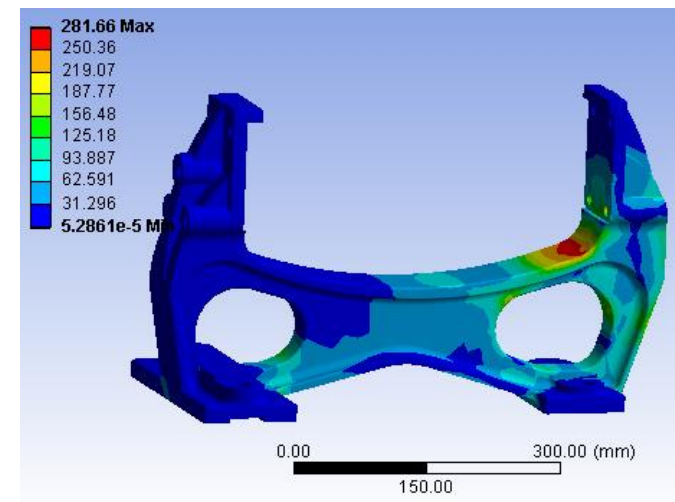

Positive Rotation

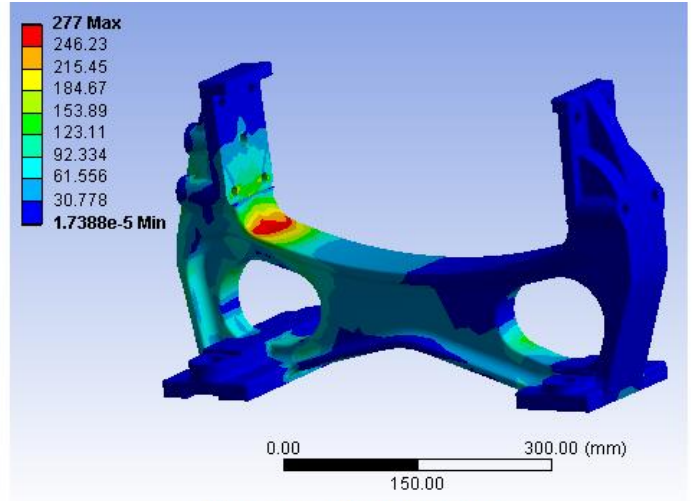

Reversed Rotation

Fig. (3). Stress Nephogram of the bracket.

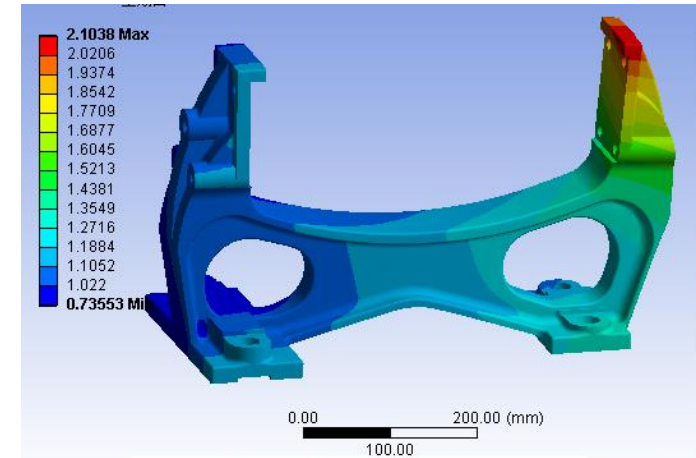

Positive Rotation

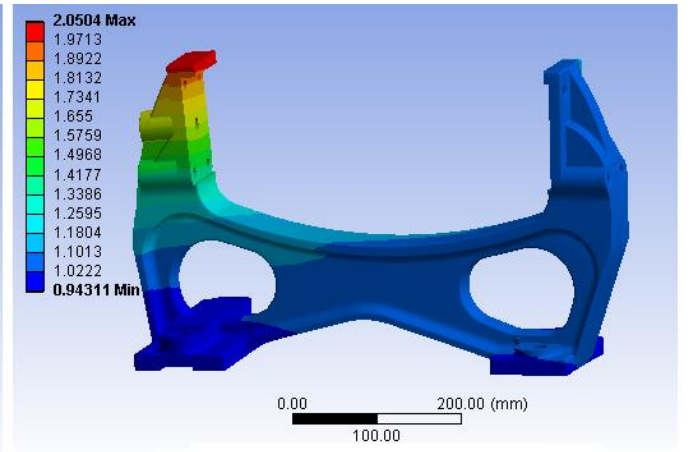

Reversed Rotation

Fig. (4). Deformation nephogram of the bracket.

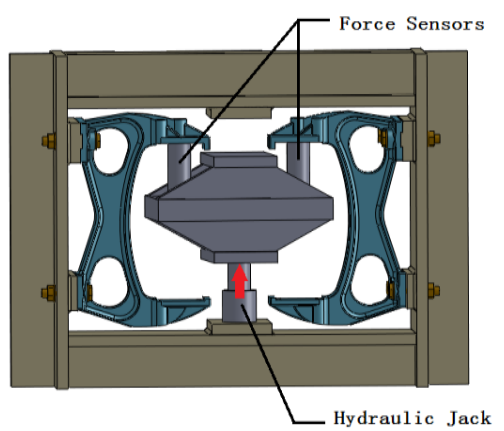

Reversed Rotation

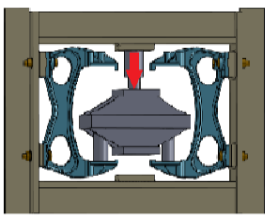

Positive Rotation

Fig. (5). Stress experiment methods.

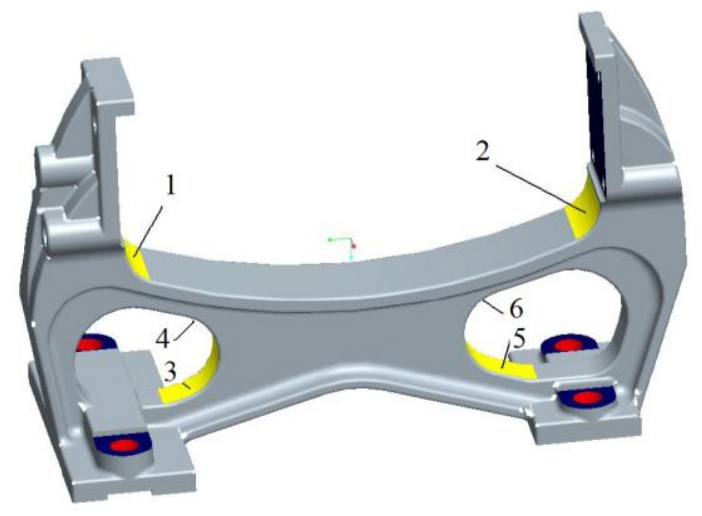

To test the bracket in the other rotating mode, just replace the hydraulic jack and the sensors as shown in Fig. (5). Fig. (8) is a photo of the experiment.

\subsection{Calculations of $45^{\circ}$ Strain Rosettes}

Fig. (9) is a diagram of a $45^{\circ}$ strain rosette. The principle strain is calculated as follows:

$$
\left.\begin{array}{l}
\max \\
\min
\end{array}\right\}=\frac{0_{0}+90}{2} \pm \sqrt{\left(\frac{0 \quad 90}{2}\right)^{2}+\left(\frac{0+90245}{2}\right)}
$$

where,

$$
\begin{aligned}
& \max \quad-- \text { maximum principle strain; } \\
& \min --- \text { minimum principle strain; } \\
& { }_{0}, \quad{ }_{45}, 90^{---s t r a i n} \text { of the strain rosette. }
\end{aligned}
$$
is:

According to Hooke's law, the maximum principle stress

$$
\max _{\max }=E \quad \max
$$

where,

$$
\max \text {---maximum principle stress; }
$$

$E$---Young's modulus.

Fig. (6). Danger points of the bracket. 

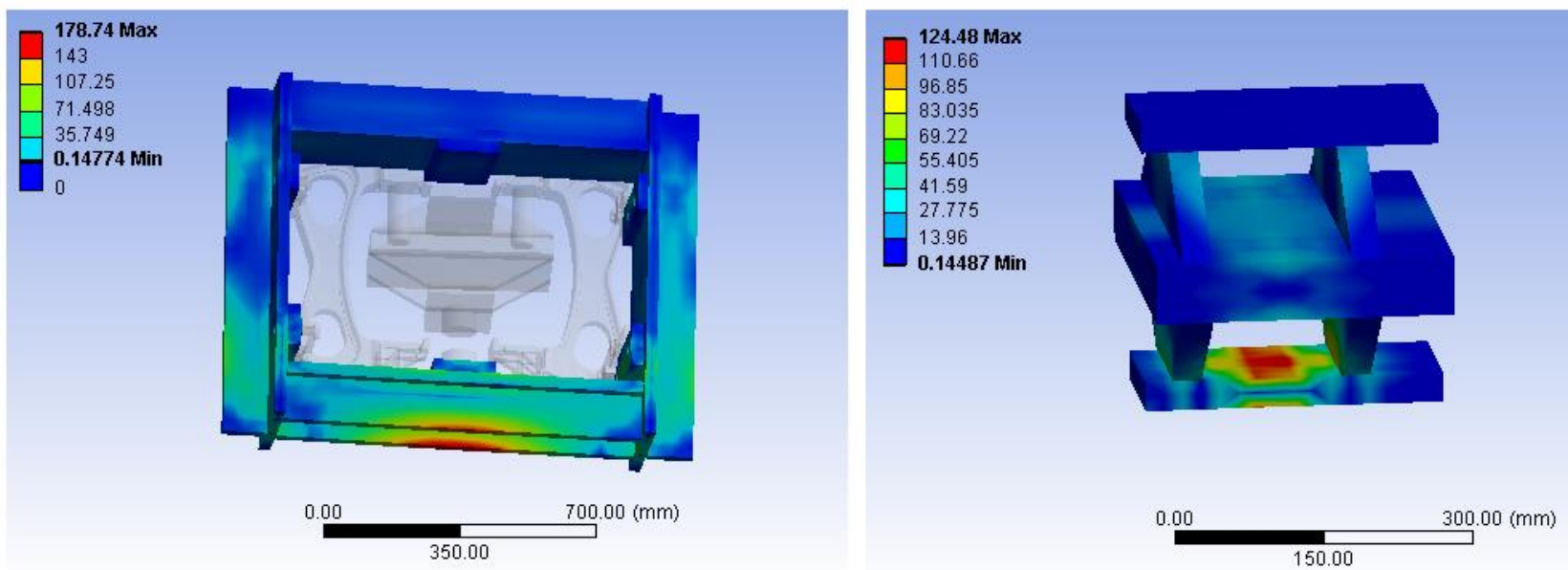

Fig. (7). Stress nephogram of the holding devices.

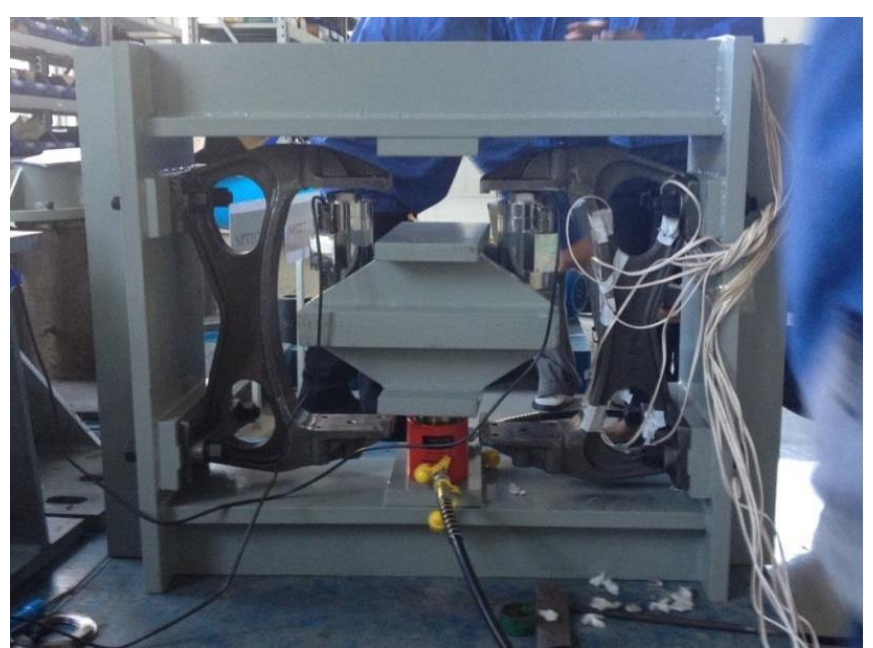

Fig. (8). Experiment scene.

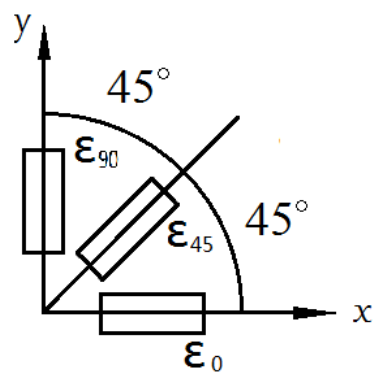

Fig. (9). $45^{\circ}$ strain rosette.

\section{SUMMARY OF FEA AND EXPERIMENTAL RESULTS}

Table 3 and Fig. (10) show the FEA and experimental stress of each measured point during positive rotation; Table 4 and Fig. (11) show the stress during reversed rotation.

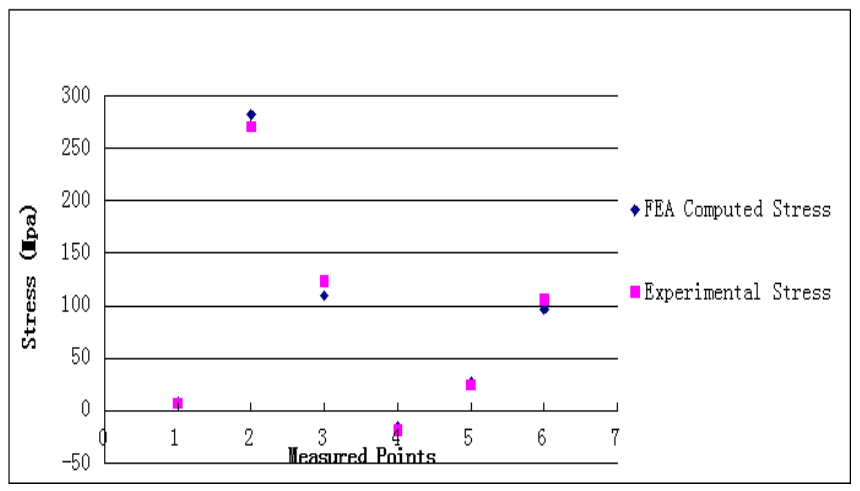

Fig. (10). Result comparison.

\section{CONCLUSION}

In this paper, a finite-element model of a $\mathrm{C}$-shaped bracket with $112 \mathrm{kN}$ load has been built for FEA uses, and a corresponding stress experiment has been performed to verify. Through the comparison of FEA and experimental results, it can be concluded that::

- It is observed that the computed values from FEA are in very good agreement with the experimental values,

Table 3. Result summary (positive).

\begin{tabular}{|c|c|c|c|c|}
\hline Measured Points & FEA Stress (MPa) & Experimental Stress (MPa) & Error Rate & Safety Factor \\
\hline \hline $\mathbf{1}$ & 7.6 & 6.9 & $10.10 \%$ \\
\hline $\mathbf{2}$ & 281.7 & 270.5 & $4.10 \%$ \\
\hline $\mathbf{3}$ & 109.4 & 123 & $-11.10 \%$ & \multicolumn{2}{c|}{2.45} \\
\hline $\mathbf{4}$ & -15.3 & -18.9 & $6.10 \%$ \\
\hline $\mathbf{5}$ & 26.4 & 24.7 & $6.80 \%$ \\
\hline
\end{tabular}


Table 4. Result summary (reversed).

\begin{tabular}{|c|c|c|c|c|}
\hline Measured Points & FEA Stress (MPa) & Experimental Stress (MPa) & Error Rate & Safety Factor \\
\hline \hline $\mathbf{1}$ & 277 & 249.7 & $10.9 \%$ & $5.7 \%$ \\
\hline $\mathbf{2}$ & -37.1 & -35.1 & $0.0 \%$ & 2.49 \\
\hline $\mathbf{3}$ & 0.4 & 0.4 & $-16.2 \%$ & 2.76 \\
\hline $\mathbf{4}$ & -48 & -57.3 & $8.1 \%$ & \\
\hline $\mathbf{5}$ & 188.4 & 174.3 & $-9.4 \%$ \\
\hline
\end{tabular}

which indicates that FEA is a reliable mothed for strength analysis.

- $\quad$ The C-shaped bracket has a maximum stress point of $270.5 \mathrm{MPa}$, which is near the corner next to the loading point. The safety factor under this condition is 2.55 , which satisfies the strength requirement.

- The maximum deformation is at the outside edge of the loading surface, with a value of $2.1 \mathrm{~mm}$, which satisfies the stiffness requirement.

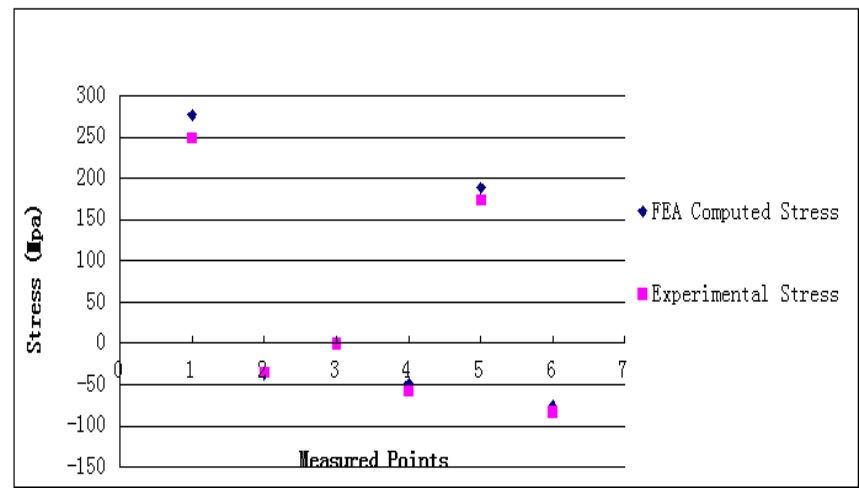

Fig. (11). Result comparison.

\section{CONFLICT OF INTEREST}

The authors confirm that this article content has no conflict of interest.

\section{ACKNOWLEDGEMENTS}

This paper was supported by the national natural science fund project (51175028), Beijing outstanding talent training projects (2010D005017000007). and the International Science \& Technology Cooperation Program of China (2014DFR70280) and BUCEA Urban Rural Construction and Management Industry Research Development Collabration Post Graduate Training Centre.

\section{REFERENCES}

[1] M. Holzapfel, and B. Wang, "Peak-performance Drives for 350km/h Latest developments in axle-mounted final drive units," High Power Converter Technology, vol. 3, pp. 34-39, Jul. 2006.

[2] Y. Chen, H.J. Zhang, Z.H. Tan, K.Z. Luo, and T. Jian, "A C-shaped bracket to hold the gearbox," C.N. Patent, 201320706626.5, Nov. 2013.

[3] A.R. Murthy, N.R. Iyer, and B.K.R. Prasad, "fracture analysis of high strength and ultra high strength concrete beams by using finite element method," Computers, Materials, \& Continua, vol. 30, no. 2, pp. 177-194, 2008.

[4] F. Yang, W.Y. Li, H.W. Li, and Y.B. Su, "Finite element analysis for gap disp disc of no-till planter," Transactions of the Chinese Society for Agricultural Machinery, vol. 41, pp. 53-55, Jun. 2010.

[5] X.M. Sun, and G.Q. Zhao, "Fake porthole extrusion die structure design and strength analysis for cantilever aluminum alloy profiles,' Journal of Mechanical Engineering, vol. 49, pp. 39-44, Dec. 2013.

[6] Z.Q. Yao, and J. Yang, "Structural strength analysis of offshore wind turbine installation vessel in the survival condition," Journal of Ship Mechanics, vol. 17, pp. 1293-1299, Dec. 2013.

[7] F. Xue, and J.H. Feng, "A Mobile Data Acquisition System and Method Based on the RFID Multi-protocol," C.N. Patent, 102110134048.8 , Nov. 2012.

(C) Yang et al.; Licensee Bentham Open.

This is an open access article licensed under the terms of the Creative Commons Attribution Non-Commercial License (http://creativecommons.org/licenses/by-nc/3.0/) which permits unrestricted, non-commercial use, distribution and reproduction in any medium, provided the work is properly cited. 